\title{
A Perplexing Case of Urinary Bladder Mass
}

\author{
Aekta Shah ${ }^{1}$ Santosh Menon ${ }^{1}$ Munita Bal ${ }^{1}$ Ganesh Bakshi ${ }^{2}$ Archi Agrawal ${ }^{3}$ Sangeeta B. Desai ${ }^{1}$
}

${ }^{1}$ Department of Pathology, Homi Bhabha National Institute, Tata Memorial Hospital, Mumbai, Maharashtra, India

2 Department of Genitourinary Surgery, Homi Bhabha National Institute, Tata Memorial Hospital, Mumbai, Maharashtra, India

${ }^{3}$ Department of Nuclear Medicine, Homi Bhabha National Institute, Tata Memorial Hospital, Mumbai, Maharashtra, India

South Asian J Cancer 2022;11(2):178-180.

Urothelial carcinoma is the commonest urinary bladder (UB) malignancy. ${ }^{1}$ However, rarely an unusual histology of a UB mass can trigger a difficult and perplexing pursuit for a correct diagnosis. Herein, we present a case of an exceptionally rare scenario presenting as a primary UB mass that after a long chase and a multitude of blind ends eventually succeeded in a fortuitous diagnosis and a fortunate outcome for the patient. Apart from turning the patient management toward the right direction, this case taught us to persevere toward a diagnosis.

A 34-year-man, occasional smoker, presented with painless hematuria for a duration of 2 months. The episodes of hematuria were intermittent, never severe enough to require a transfusion. General physical examination was unremarkable, and all laboratory investigations were within normal limits. Urine cytology was negative for high-grade urothelial carcinoma. Computed tomography scans revealed a $2.9 \times 3.5$ $\times 2.6 \mathrm{~cm}$ ill-defined enhancing polypoidal soft tissue lesion in the left posterolateral wall of the UB with focal perivesical extension. Kidneys, prostate, and seminal vesicles were unremarkable. Cystoscopy revealed a large flat, erythematous area measuring $5 \times 4 \mathrm{~cm}$ in the UB. With a clinical diagnosis of urothelial carcinoma, a transurethral resection of bladder tumor was performed.

Microscopy revealed a tumor with a conspicuous nested pattern expanding the lamina propria ( - Fig. 1A, B). The neoplastic cells were monomorphic and possessed bland nuclei with fine chromatin and inconspicuous nucleoli. Mitosis was inconspicuous and necrosis was absent. Considering differential diagnoses of paraganglioma and a nested variant of urothelial carcinoma, an immunohistochemistry panel

\section{DOI https://doi.org/10.1055/s-0041-1731908 ISSN 2278-330X}

How to cite this article: Shah A, Menon S, Bal M, et al. A Perplexing Case of Urinary Bladder Mass South Asian J Cancer 2022;11(2): South Asian J Cancer 2022;11(2):178-179.
Address for correspondence Santosh Menon, MD, Department of Pathology, Tata Memorial Centre, Homi Bhabha National Institute, Mumbai 400012, Maharashtra, India (e-mail: mensantosh@gmail.com).

comprising AE1/AE3, p63, high-molecular weight cytokeratin, GATA3, synaptophysin and chromogranin was used. All markers were negative except for AE1/AE3, excluding neuroendocrine neoplasm or urothelial malignancy. Next step led to adding CK7, CK20, CDX2, PSA, and AMACR to exclude an infiltrating rectal or prostatic primary. All were negative barring a diffuse CK7. Adding calretinin and inhibin to rule out a mesothelial neoplasm or a sex-cord stromal tumor, respectively turned out to be futile as tumor remained

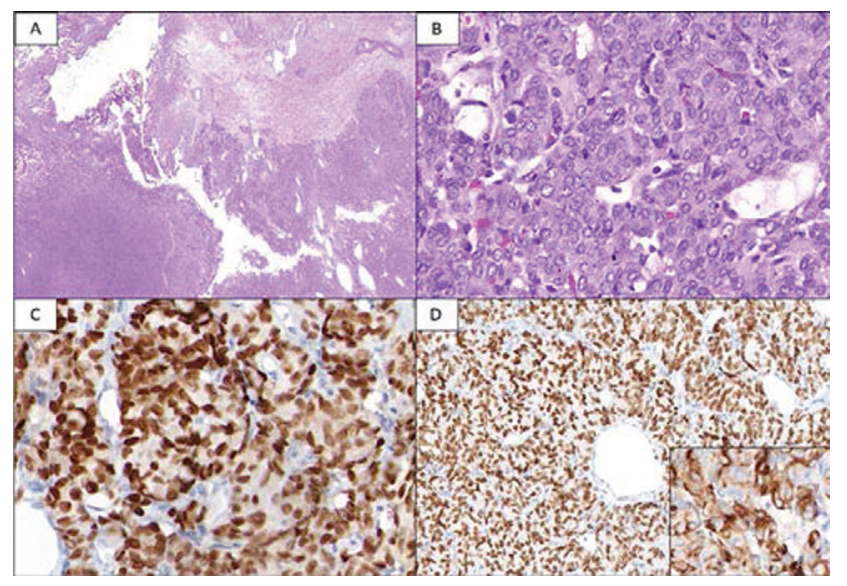

Fig. $1 \mathrm{H}$ and $\mathrm{E}(\mathrm{A}, \mathrm{B})$ and IHC (C,D) Photomicrographs of Transurethral resection of urinary bladder (TURBT). (A) Tumor in the bladder involving the lamina propria $(\times 100)$. (B) Morphology is similar to paraganglioma/nested variant of urothelial carcinoma $(\times 200)$. (C) PAX8 is strongly and diffusely positive $(\times 400)$. (D) TTF1 is strongly and diffusely positive $(\times 200)$, diffuse thyroglobulin positivity (inset).

(c) 2022. MedIntel Services Pvt Ltd. All rights reserved.

This is an open access article published by Thieme under the terms of the Creative Commons Attribution-NonDerivative-NonCommercial-License, permitting copying and reproduction so long as the original work is given appropriate credit. Contents may not be used for commercial purposes, or adapted, remixed, transformed or built upon. (https://creativecommons.org/licenses/by-nc-nd/ 4.0/)

Thieme Medical and Scientific Publishers Pvt. Ltd., A-12, 2nd Floor, Sector 2, Noida-201301 UP, India 


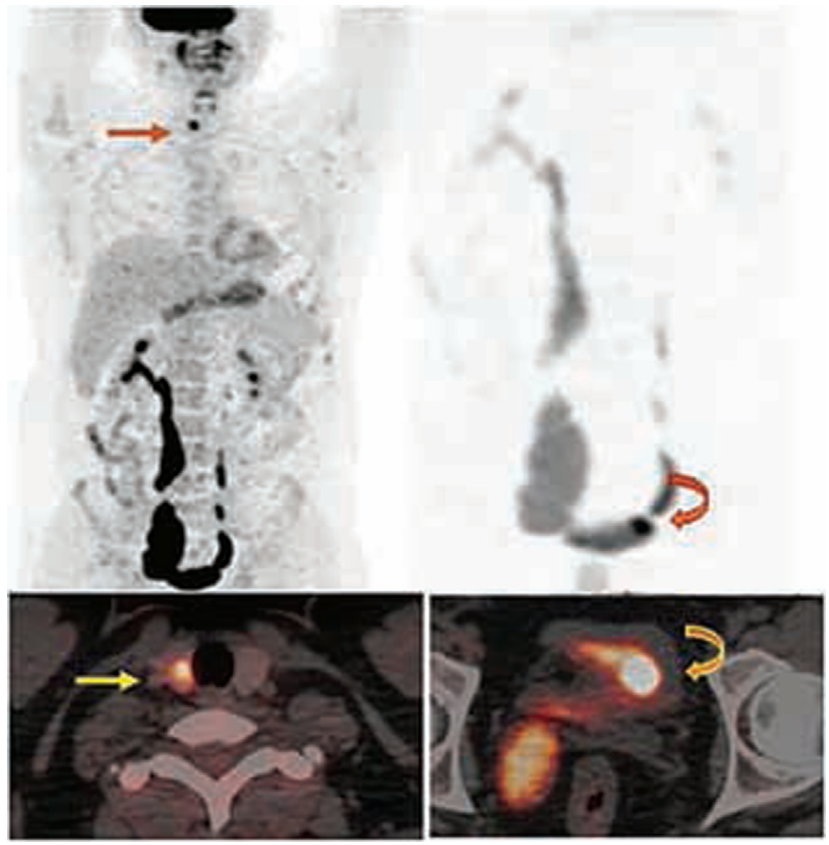

Fig. 2 FDG PET revealed hypermetabolic right thyroid nodule (straight arrow) and hypermetabolic bladder mass (curved arrow).

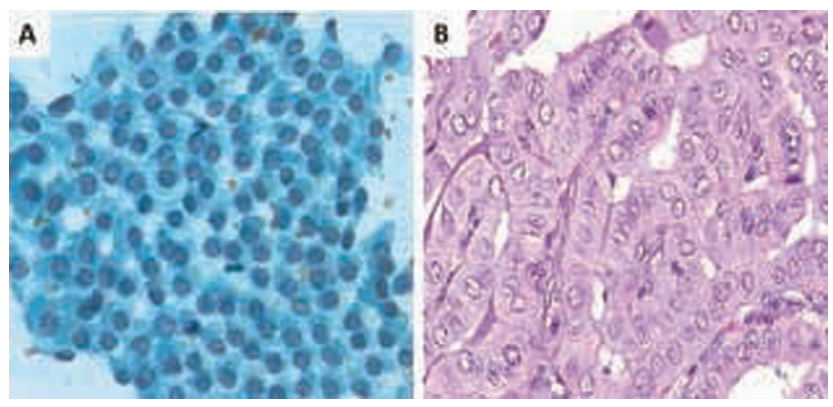

Fig. 3 Papanicolaou stain (A) FNAC of right thyroid nodule shows papillaroid fragments with overlapping, grooving and intranuclear inclusions $(\times 400)$. $\mathrm{H}$ and $\mathrm{E}$ stain $(\mathrm{B})$ Thyroidectomy: Papillary thyroid carcinoma $(\times 400)$.

negative. Exhausting nearly all common and uncommon diagnoses for this in this location, prompted a blind gamble with PAX8 which serendipitously marked the tumor nuclei diffusely and strongly. This coupled with the differentiated appearance of the tumor narrowed the possibilities considerably and was followed up with TTF1 and thyroglobulin which were strong and diffusely positive, confirming a surprise primary of thyroid origin ( - Fig. 1C, D). A complete radiological evaluation of the thyroid and a positron emission tomography/computed tomography (PET/CT) was ordered, which revealed hypermetabolic right thyroid nodule (SUV max of 15.65) in addition to the hyper-metabolic UB mass ( - Fig. 2). Ultrasonography of thyroid revealed a solid hypo-echoic nodule, measuring $12 \times$ $13 \mathrm{~mm}$, in the right lobe. Fine needle aspiration yielded a diagnosis of papillary thyroid carcinoma (PTC) (Bethesda category $\mathrm{VI}$ ) ( $\sim$ Fig. 3A). Total thyroidectomy was performed; a gray-white firm tumor measuring $2.5 \times 1.8 \times 1.5 \mathrm{~cm}$ was located in the lower part of right lobe. Microscopy revealed histology of tall cell variant of PTC ( - Fig. 3B) with extrathyroidal extension and negative regional lymph nodes. Patient was treated with adjuvant radioactive iodine (RAI). There was no residual disease in the thyroid bed or in the UB on cystoscopy at last follow-up of 25 months duration.

Knowledge of metastatic involvement of UB by thyroid carcinoma is important as it entails an entirely different management comprising RAI following thyroidectomy instead of cystectomy for a primary UB malignancy. Only two cases of solitary metastasis from thyroid to UB have been reported previously both of which had a known history of thyroidectomy for differentiated thyroid carcinoma 14 years and 5 months earlier, respectively. ${ }^{2,3}$ Another case of metastasis to UB is documented wherein UB involvement was a part of disseminated thyroid malignancy. ${ }^{4}$ Present case was novel in its clinical presentation and therefore represents the first case of PTC (tall cell variant) appearing as a UB malignancy without clinical disease elsewhere. In addition to the extraordinary rarity of the case, this report exemplifies the uncompromising approach to pursue an accurate diagnosis that forms the foundation of correct management.

Conflict of Interest

None declared.

\section{References}

1 Grignon DJ, Al-Ahmadie H, Algaba F, et al. Infiltrating urothelial carcinoma. In: Moch H, ed. WHO Classification of Tumours of the Urinary System and Male Genital Organs. 4th edLyon:: IARC Press; 2016:81-98

2 Ando T, Matsuo Y, Ikeoka T, et al. Gross hematuria and bladder tumor in a patient with advanced thyroid papillary carcinoma. Case Rep Endocrinol 2013;2013:585781

3 Grivas N, Housianitis Z, Doukas M, Stavropoulos NE. Follicular thyroid cancer metastasis to the urinary bladder: report of a case and review of the literature. Case Rep Urol 2012;2012:178915

4 Pepe P, Fraggetta F, Galia A, Grasso G, Aragona F. Hematuria as the presenting symptom of a metastatic thyroid cancer. Urol Int 2006; 76(01):89-90 